\title{
HPV Vaccine Acceptance Among Mid-Adult Women
}

\author{
Daron G. Ferris, MD, Jennifer L. Waller, PhD, Ashley Owen, and Jozette Smith
}

Objective: To determine correlates of human papillomavirus (HPV) vaccine acceptance in mid-adult women.

Methods: A convenience sample of 472 mid-adult women completed a 2-part, 69-item survey that included demographic, knowledge, and behavioral variables as potential correlates of vaccine acceptance. Univariable and multivariable logistic regression models were used to identify correlates for vaccine acceptance.

Results: Mid-adult women who received the HPV vaccine were more likely to be younger than 55 years $(P<.001)$; have had an abnormal Papanicolaou test (odds ratio [OR], 2.15; 95\% CI, 1.18-3.92); understand that HPV causes cervical cancer (OR, 2.39; 95\% CI, 1.08-5.30); feel at risk for HPV infection (OR, 2.14; 95\% CI, 1.00-4.57), and feel it is important for their partner (OR, 25.20; 95\% CI, 9.66-65.72) and children (OR, 3.54; CI, 0.51-24.56) to get the HPV vaccine. Monogamous mid-adult women (OR, 0.46; 95\% CI, 0.21-1.00); women who did not want any vaccines $(\mathrm{OR}, 0.26$; $95 \% \mathrm{CI}, 0.07-$ $0.92)$; and women who felt it was too late to get the vaccine $(\mathrm{OR}, 0.18 ; 95 \% \mathrm{CI}, 0.08-0.44)$ were less likely to want the HPV vaccine.

Conclusions: These clinical predictors of HPV vaccine acceptance will help clinicians recognize midadult women who may be more receptive to vaccination. (J Am Board Fam Med 2008;21:31-37.)

Primary cervical cancer prevention has become a reality with the availability of the first prophylactic human papillomavirus (HPV) vaccine (Gardasil, Merck and Co., Inc, Whitehouse Station, NJ). The quadrivalent $\mathrm{L} 1$ virus-like particle $\mathrm{HPV}$ vaccine is expected to prevent $70 \%$ of cervical cancer caused by oncogenic HPV types 16 and 18 , and $90 \%$ of genital warts caused by nononcogenic HPV types 6 and 11. When administered to adolescent and young adult women it was $100 \%$ effective in preventing true cancer precursors of the lower genital tract caused by the $4 \mathrm{HPV}$ types included in the vaccine, provided women received all 3 doses (at 0 , 2, and 6 months), and were HPV DNA negative to

This article was externally peer reviewed.

Submitted 17 April 2007; revised 2 July 2007; accepted 3 July 2007.

From the Gynecologic Prevention Center (DGF); the Department of Family Medicine and the Department of Obstetrics and Gynecology (DGF); the Department of Biostatistics (JLW), Medical College of Georgia (AO); and Paine College (JS), Augusta, Georgia.

Funding: none.

Conflict of interest: Dr. Ferris is a clinical investigator and consultant for Merck and Co., Inc., and GlaxoSmithKline. $\mathrm{He}$ is also a speaker for Merck and Co., Inc.

Corresponding author: Daron G. Ferris, MD, Department of Family Medicine, Medical College of Georgia, 1423 Harper Street, HH-105, Augusta, GA, 30912 (E-mail: agerman@mcg.edu). the 4 HPV types at day 0 and remained HPV DNA negative to the 4 HPV types until after completion of the third dose. ${ }^{1}$ This amazingly robust result can be expected when the vaccine is administrated to children, adolescents, and young women (ages 9 to 26 years) who are not yet sexually active.

However, mid-adult women ( $\geq 27$ years old) have expressed a keen interest in also receiving the HPV vaccine. ${ }^{2}$ These women want the vaccine to stay healthy, to prevent cervical cancer and genital warts, and because of a fear of cervical cancer and genital warts. $^{2,3}$ Although many women in this age group are no longer considered sexually naïve, there may be good rationale for vaccinating these more mature and informed women. It is very unlikely that most mid-adult women have been previously exposed to all 4 HPV types from which the vaccine affords protection. Based on an acceptable safety profile for children and younger women, one could assume the vaccine will also convey a comparable safety profile for older women. ${ }^{1}$ Although neutralizing antibody levels are expected to be lower in mid-adult women, we would expect ample neutralizing antibody titers to provide protection based on the robust immune response demonstrated in the younger population. Currently, safety, immunogenicity, and efficacy data are lack- 
Table 1. Demographic Variables of Women in the Univariable Logistic Regression Models Wanting to Get the Human Papillomavirus Vaccine After Intervention

\begin{tabular}{|c|c|c|c|c|c|}
\hline Demographic Variables & Level & Odds Ratio & $95 \% \mathrm{CI}$ & Wald $\chi^{2}$ & $P$ \\
\hline \multirow[t]{7}{*}{ Age (years) } & & & & 21.43 & .0015 \\
\hline & $25-29$ vs $55+$ & 11.39 & $3.58-6.22$ & & \\
\hline & $30-34$ vs $55+$ & 8.8 & $2.53-30.57$ & & \\
\hline & $35-39$ vs $55+$ & 10.15 & $2.88-35.83$ & & \\
\hline & $40-44$ vs $55+$ & 12.37 & $3.20-47.83$ & & \\
\hline & $45-49$ vs $55+$ & 6.68 & $1.86-23.99$ & & \\
\hline & $50-54$ vs $55+$ & 4.63 & $1.32-16.26$ & & \\
\hline \multirow[t]{7}{*}{ Family income $(\$)$} & & & & 12.32 & .055 \\
\hline & $10,000-19,000$ vs $>100,000$ & 9.60 & $2.41-38.22$ & & \\
\hline & $19,001-29,000$ vs $>100,000$ & 2.51 & $0.99-6.37$ & & \\
\hline & $29,001-39,000$ vs $>100,000$ & 2.93 & $1.13-7.62$ & & \\
\hline & $39,001-50,000$ vs $>100,000$ & 2.16 & $0.91-5.14$ & & \\
\hline & $50,001-100,000$ vs $>100,000$ & 1.95 & $0.89-4.24$ & & \\
\hline & $<10,000$ vs $>100,000$ & 2.29 & $0.72-7.30$ & & \\
\hline Medical insurance & Yes vs no & 0.37 & $0.16-0.86$ & 5.37 & .02 \\
\hline \multirow[t]{5}{*}{ Marital status } & & & & 10.75 & .03 \\
\hline & Married vs widowed & 0.93 & $0.18-4.77$ & & \\
\hline & Divorced/separated vs widowed & 1.86 & $0.33-10.45$ & & \\
\hline & Live with partner vs widowed & 2.50 & $0.35-18.03$ & & \\
\hline & Single vs widowed & 2.16 & $0.41-11.46$ & & \\
\hline Monogamous & Yes vs no & 0.54 & $0.27-1.06$ & 3.21 & .07 \\
\hline Age at first sexual intercourse (years) & & 0.89 & $0.82-0.97$ & 6.81 & .009 \\
\hline Vaginal sex & Yes vs no & 2.35 & $1.02-5.37$ & 4.06 & .04 \\
\hline \multirow[t]{3}{*}{ Birth control } & & & & 4.50 & .11 \\
\hline & Barrier vs abstinence & 1.25 & $0.35-4.94$ & & \\
\hline & Non-barrier vs abstinence & 0.60 & $0.18-2.04$ & & \\
\hline STI status & Any STI vs no STI & 2.10 & $1.18-3.74$ & 6.35 & .01 \\
\hline \multirow[t]{3}{*}{ Abnormal Pap test } & & & & 6.80 & .03 \\
\hline & DK vs no & 1.39 & $0.48-4.06$ & & \\
\hline & Yes vs no & 2.06 & $1.20-3.57$ & & \\
\hline Age at first Pap test & & 0.93 & $0.85-1.01$ & 3.23 & .07 \\
\hline Have had a mammogram & Yes vs no & 0.49 & $0.30-0.81$ & 7.91 & .005 \\
\hline
\end{tabular}

Referent groups are listed second for all levels.

HPV, human papillomavirus; STI, sexually transmitted infection; DK, does not know if they have ever had an abnormal Pap test.

ing for mid-adult women. Ongoing clinical trials will provide these important data for mid-adult women in the next few years.

As opposed to an established and recommended HPV vaccination program for children and young women, a "catch-up" vaccination program for midadult women has not yet been implemented. Many mid-adult women are interested in receiving the HPV vaccine, but others are not., Identified barriers to vaccine acceptance include being married, cost, and possible perception of being promiscuous if they receive the HPV vaccine. ${ }^{3}$ To date, little is known about predictors of HPV vaccination preference by an older group of women. The purpose of these analyses were to determine correlates of acceptance of the HPV vaccine by mid-adult women.

\section{Methods}

\section{Subject Population}

We recruited a convenience sample of mid-adult women ( $\geq 25$ years old) from the community and medical clinics located in 3 southern US cities (Atlanta and Augusta, Georgia, and San Antonio, Texas) during the summer of 2006. An arbitrary inclusion limit of women 25 years or older was considered, and this range was selected before the FDA approval for current vaccination of women up 
Table 2. Knowledge and Behaviors Before Intervention of Women in Univariable Logistic Regression Models Wanting to Get the Human Papillomavirus Vaccine After Intervention

\begin{tabular}{|c|c|c|c|c|c|}
\hline Variables & Levels & Odds Ratio & $95 \% \mathrm{CI}$ & Wald $\chi^{2}$ & $P$ \\
\hline \multirow[t]{3}{*}{ Important to get vaccines } & & & & 5.63 & .06 \\
\hline & Important vs not important & 4.48 & $1.05-19.14$ & & \\
\hline & Neutral vs not important & 2.50 & $0.56-11.23$ & & \\
\hline HPV causes cancer & Yes vs no & 2.47 & $1.13-5.43$ & 5.10 & .02 \\
\hline At risk for HPV & Yes vs no & 2.21 & $1.04-4.68$ & 4.28 & .04 \\
\hline Know what HPV vaccine is for & Yes vs no & 1.94 & $0.93-4.03$ & 3.14 & .08 \\
\hline \multirow[t]{3}{*}{ Important to get HPV vaccine } & & & & 20.81 & $<.0001$ \\
\hline & Important vs not important & 40.61 & $8.15-202.42$ & & \\
\hline & Neutral vs not important & 19.80 & $4.09-95.96$ & & \\
\hline
\end{tabular}

HPV, human papillomavirus.

to age 26. Other inclusion criteria were an ability to read and write in English. The Medical College of Georgia Human Assurance Committee approved this study (HAC file \#06-04-276).

\section{Study Design and Instrument}

Complete details of the HPV vaccine acceptance questionnaire for mid-adult women have been published previously. ${ }^{2}$ Survey development began with focus group input, followed by a pretrial pilot of the draft survey. Subjects completed a 46-question initial (pre-intervention) survey, read a 1-page educational pamphlet about HPV and HPV vaccines, then completed a 23-question postintervention survey. Certain questions included in these surveys allowed for assessment of demographic, knowledge, and behavioral variables as potential correlates of HPV vaccine acceptance among mid-adult women. The pre-intervention survey included demographic data, sexual history, Papanicolaou test and sexually transmitted infection history, lifestyle practices, knowledge about vaccines, cervical cancer, and attitudes about HPV vaccines. The postintervention survey assessed subjects' attitudes, knowledge, and acceptance of the HPV vaccines. Each survey included multiple choice, true/false, open-ended, rank preference by order, and multiple response questions. The same HPV educational pamphlet and portions of the survey were used in a previous study of parents' acceptance of an HPV vaccine for their children. ${ }^{4}$

\section{Statistical Analyses}

Each potential correlate of wanting to get the HPV vaccine after intervention was examined first using $\chi^{2}$ tests or $t$ tests. There were 3 sets of correlates that were examined: (1) demographic variables, (2) knowledge and behaviors before intervention, and (3) knowledge and behaviors after intervention. Those variables statistically significant in the $\chi^{2}$ or $t$ tests were entered into a univariable logistic regression model and the odds ratio, $95 \%$ confidence interval for the odds ratio, and statistical significance were determined. For each set of models (demographic, pre-intervention, or postintervention), those variables statistically significant in the univariable models were placed in a multivariable logistic regression model. A backwards, stepwise elimination procedure was used on each model set to arrive at a final multivariable logistic regression model for the set. All statistical significance was assessed using an $\alpha$-level of 0.05 and all statistical analyses were performed using SAS version 9.1.3 (SAS Institute, Inc., Cary, NC).

\section{Results}

\section{Demographic Data}

A total of 675 women were asked to participate in the study. Complete demographic data for the 472 participating subjects have been previously published. ${ }^{2}$ Approximately half were white, married, and younger than 35 years old. Although two thirds had a college degree, only half had a family income $\geq \$ 39,001 ; 13 \%$ were uninsured. Nearly three quarters were in a monogamous sexual relationship, but subjects reported a mean lifetime number of sexual partners of 6.3 (SD, 7.7). Twelve percent had a history of cervical neoplasia. 
Table 3. Knowledge and Behaviors After Intervention in Women in Univariable Logistic Regression Models Wanting to Get the Human Papillomavirus Vaccine After Intervention

\begin{tabular}{|c|c|c|c|c|c|}
\hline Variable & Levels & Odds Ratio & $95 \% \mathrm{CI}$ & Wald $\chi^{2}$ & $P$ \\
\hline $\begin{array}{l}\text { Intervention information changed mind about } \\
\text { HPV vaccine }\end{array}$ & Yes vs no & 3.52 & $2.06-6.00$ & 21.35 & $<.0001$ \\
\hline At risk for $\mathrm{HPV}$ & Yes vs no & 25.81 & $10.07-66.17$ & 45.82 & $<.0001$ \\
\hline \multirow[t]{3}{*}{ Would get any vaccine } & & & & 27.87 & $<.0001$ \\
\hline & No vaccine vs STI vaccine & 0.12 & $0.04-0.32$ & & \\
\hline & Other vaccine vs STI vaccine & 0.07 & $0.02-0.32$ & & \\
\hline \multirow[t]{3}{*}{ Important for partner to get HPV vaccine } & & & & 77.04 & $<.0001$ \\
\hline & Important vs not important & 120.17 & $40.83-353.68$ & & \\
\hline & Neutral vs not important & 11.02 & $5.01-24.24$ & & \\
\hline \multirow[t]{3}{*}{ Important for children to get $\mathrm{HPV}$ vaccine } & & & & 38.48 & $<.0001$ \\
\hline & Important vs not important & 18.19 & $3.98-83.19$ & & \\
\hline & Neutral vs not important & 4.06 & $0.86-19.27$ & & \\
\hline \multirow[t]{3}{*}{ Pap smears important } & & & & 8.98 & .01 \\
\hline & Important vs not important & 1.77 & $0.11-28.55$ & & \\
\hline & Neutral vs not important & 0.25 & $0.01-5.26$ & & \\
\hline Too late to get HPV vaccine & Yes vs no & 0.14 & $0.07-0.27$ & 31.13 & $<.0001$ \\
\hline \multicolumn{6}{|l|}{ More inclined to get HPV vaccine if... } \\
\hline Recommended by family member & Yes vs no & 4.27 & $1.84-9.88$ & 11.44 & .0007 \\
\hline Recommended by nurse & Yes vs no & 3.51 & $1.74-7.07$ & 12.33 & .0004 \\
\hline Paid for by insurance & Yes vs no & 3.22 & $1.97-5.25$ & 21.83 & $<.0001$ \\
\hline \multirow{5}{*}{$\begin{array}{l}\text { Amount would pay for HPV vaccine to prevent } \\
\text { cervical cancer and genital warts }(\$)\end{array}$} & & & & 15.55 & .004 \\
\hline & $101-200$ vs none & 6.23 & $2.17-17.90$ & & \\
\hline & $201+$ vs none & 4.11 & $1.72-9.80$ & & \\
\hline & $51-100$ vs none & 3.76 & $1.63-8.70$ & & \\
\hline & $<50$ vs none & 2.59 & $1.14-5.86$ & & \\
\hline HPV causes cervical cancer & Yes vs no & 2.20 & $1.30-3.72$ & 8.71 & .003 \\
\hline HPV can prevent genital warts & Yes vs no & 1.76 & $0.95-3.28$ & 3.19 & .07 \\
\hline
\end{tabular}

HPV, human papillomavirus; STI, sexually transmitted infection.

\section{Univariable Model Analyses}

The univariable model analyses included complete survey responses from 280 patients for demographic variables, 201 patients for knowledge and behaviors before intervention, and 302 patients for knowledge and behaviors after intervention. These 3 univariable models are presented in Tables 1 to 3 , respectively.

\section{Multivariable Demographic Analyses}

The final multivariable models for the demographic variables, knowledge, and behaviors before intervention and knowledge and behaviors after intervention are presented in Table 4. The final demographic model contained age, being in a monogamous relationship, and previous abnormal Papanicolaou tests. Compared with women 55 years of age and older, every other age group of mid-adult women was more likely to want to get the HPV vaccine after interven- tion $\left(\chi^{2}, 21.7 ; P=.001\right)$. Those who were in monogamous relationships were less likely to want to get the HPV vaccine after intervention (odds ratio [OR], 0.46; 95\% CI, 0.21-1.00). Subjects who had a previous history of an abnormal Papanicolaou test were more likely (OR, 2.15; 95\% CI, 1.18-3.92) to want to get the HPV vaccine after intervention than those who had not had an abnormal Papanicolaou test.

\section{Multivariable Knowledge and Behavior at Analyses Before Intervention}

For knowledge and behaviors before intervention, knowledge that HPV causes cervical cancer and knowledge of being at risk for HPV infection were significant correlates of wanting the HPV vaccine after intervention. Women who knew that HPV causes cancer before intervention were more likely (OR, 2.39; 95\% CI, 1.08-5.30) to want the HPV 
Table 4. Final Multivariable Logistic Regression Models for Wanting to Get the Human Papillomavirus Vaccine After Intervention: Demographics, Knowledge, and Behaviors Before Intervention and Knowledge and Behaviors After Intervention

\begin{tabular}{|c|c|c|c|c|c|}
\hline Variables & Level & Odds Ratio & $95 \% \mathrm{CI}$ & Wald $\chi^{2}$ & $P$ \\
\hline \multicolumn{6}{|l|}{ Demographic } \\
\hline \multirow[t]{7}{*}{ Age (years) } & & & & 21.66 & 0.001 \\
\hline & $25-29$ vs $55+$ & 11.62 & $3.60-37.51$ & & \\
\hline & $30-34$ vs $55+$ & 8.89 & $2.50-31.61$ & & \\
\hline & $35-39$ vs $55+$ & 9.62 & $2.66-34.79$ & & \\
\hline & $40-44$ vs $55+$ & 11.63 & $2.90-46.62$ & & \\
\hline & $45-49$ vs $55+$ & 7.53 & $2.05-27.68$ & & \\
\hline & $50-54$ vs $55+$ & 4.00 & $1.11-14.39$ & & \\
\hline Monogamous & Yes vs no & 0.46 & $0.21-1.00$ & 3.85 & 0.05 \\
\hline \multirow[t]{3}{*}{ Abnormal Pap test } & & & & 6.84 & 0.03 \\
\hline & DK vs no & 0.79 & $0.24-2.55$ & & \\
\hline & Yes vs no & 2.15 & $1.18-3.92$ & & \\
\hline \multicolumn{6}{|l|}{ Knowledge and Behaviors Pre-Intervention } \\
\hline HPV causes cancer & Yes vs no & 2.39 & $1.08-5.30$ & 4.59 & 0.03 \\
\hline At risk for HPV & Yes vs no & 2.14 & $1.00-4.57$ & 3.88 & 0.05 \\
\hline \multicolumn{6}{|l|}{ Knowledge and Behaviors Post-Intervention } \\
\hline \multirow[t]{3}{*}{ Would get any vaccine } & & & & 7.32 & 0.03 \\
\hline & No vaccine vs STI vaccine & 0.26 & $0.07-0.92$ & & \\
\hline & Other vaccine vs STI vaccine & 0.22 & $0.04-1.10$ & & \\
\hline \multirow[t]{3}{*}{$\begin{array}{l}\text { Important for partner to get HPV } \\
\text { vaccine }\end{array}$} & & & & 47.92 & $<0.0001$ \\
\hline & Important vs not important & 25.20 & $9.66-65.72$ & & \\
\hline & Neutral vs not important & 14.44 & $5.83-35.79$ & & \\
\hline \multirow[t]{3}{*}{ Important for child to get HPV vaccine } & & & & 10.46 & 0.005 \\
\hline & Important vs not important & 3.54 & $0.51-24.56$ & & \\
\hline & Neutral vs not important & 1.04 & $0.15-7.45$ & & \\
\hline Too late to get HPV vaccine & Yes vs no & 0.18 & $0.08-0.44$ & 14.76 & 0.0001 \\
\hline
\end{tabular}

HPV, human papillomavirus; STI, sexually transmitted infection; DK, does not know if they havevever had an abnormal Pap test.

vaccine after intervention than were women who did not know that HPV causes cancer. Mid-adult women who knew they were at risk of HPV infection before intervention were more likely (OR, 2.14; 95\% CI, 1.00-4.57) to want the HPV vaccine after intervention than were women who did not know they were at risk of getting HPV.

\section{Multivariable Knowledge and Behavior Analyses After Intervention}

The multivariable model for knowledge and behaviors after intervention that were significant correlates of wanting to get the HPV vaccine after intervention included wanting to get any type of vaccine, the importance of the patient's partner getting the HPV vaccine, the importance of the patient's children getting the HPV vaccine, and it being too late to get the HPV vaccine. Mid-adult women who did not want to get any type of vaccine were less likely (OR, 0.26; 95\% CI, 0.07-0.92) to want the HPV vaccine than women who wanted to get any vaccine to prevent sexually transmitted infections. Women who felt it was important (OR, 25.20; 95\% CI, 9.66-65.72) for their partner to get the HPV vaccine or who were neutral (OR, 14.44; 95\% CI, 5.83-35.79) about their partner getting the vaccine were more likely to want to get the HPV vaccine than women who felt it was not important for their partner to get the HPV vaccine. Women who felt it was important (OR, 3.54; 95\% CI, 0.51-24.56) for their children to get the HPV vaccine or who were neutral (OR, 1.04; 95\% CI, $0.15-7.45$ ) about their children getting the vaccine were more likely to want to get the HPV vaccine than were women who felt it was not important for their children to get the HPV vaccine. Finally, 
women who felt it was too late for them to get the HPV vaccine were less likely (OR, 0.18; 95\% CI, $0.08-0.44)$ to want the HPV vaccine than midadult women who did not feel it was too late.

\section{Discussion}

We were able to determine several important independent correlates of HPV vaccine acceptance by mid-adult women. A few might be considered useful clinical predictors: younger than 55 years of age and a history of an abnormal Papanicolaou test. This information should be readily available and included in any patient's medical record. Our study demonstrated a positive age correlation for HPV vaccine acceptance even for women 45 to 49 years old (OR, 7.5; 95\% CI, 2.05-27.68). Whether women older than age 55 would benefit from receiving the HPV vaccine is unknown, and it is unlikely a clinical trial will ever address this point. Although off-label administration of the HPV vaccine is already occurring for women older than 26 years, health care providers will need to be prepared to vaccinate more "catch-up" mid-adult women if the Food and Drug Administration approves an extended age indication in a few years. Mid-adult women with a history of an abnormal Papanicolaou test have various motives for wanting the vaccine. ${ }^{2}$ From a medical perspective, there are compelling reasons to vaccinate these mid-adult women who have demonstrated immunologic vulnerability. A multivalent vaccine affords future protection against neoplasia caused by all or other HPV types included in the vaccine, depending on previous HPV exposure. This primary protection is especially important for women who are unable to prevent persistent HPV infections and ensuing neoplasia.

Mid-adult women opposed to all vaccines were not swayed by the attributes of the HPV vaccine when compared with women who wanted to receive any vaccine that would protect them from a sexually transmitted infection. A small segment of the population refuses any vaccines for a variety of reasons. ${ }^{5}$ Exemption from school-based immunization requirements are granted for religious and personal beliefs. ${ }^{6}$ Because the HPV vaccine will not be mandated for mid-adult women, universal coverage is unlikely, which is the case with most other vaccines. Health care providers know who these nonreceptive women are in their practices. Perhaps an Food and Drug Administration-approved HPV vaccine should be offered to these mid-adult women in the future if it is proven safe and efficacious for this older population.

The knowledge that HPV causes cervical cancer and the knowledge of being at risk for HPV infection were positive correlates of HPV vaccine acceptance among mid-adult women. These results reinforce the importance of patient education. As such, mid-adult women who are aware of these potential risks will be more interested in being vaccinated. Unfortunately, HPV is not one of the more readily recognized sexually transmitted infections. ${ }^{3,7,8}$ However, many health care providers are sufficiently knowledgeable about the risks of HPV to appropriately convey this information to their patients. Timely education may help minimize the cost, morbidity, and mortality associated with HPV-related neoplasias.

Believing that it is important for their partner and children to receive the vaccine were also positive predictors of HPV vaccine acceptance by midadult women. This was particularly true (OR, 25.2; 95\% CI, 9.66-65.72) for the former. Although the Food and Drug Administration has not approved the HPV vaccine for use in men yet, many from our group of receptive mid-adult women considered vaccination of their sexual partner(s) to be very important. Such a vaccination strategy would offer protection to their partner, but would also indirectly help protect them from HPV infection. Midadult mothers who want their children to receive the vaccine are also likely to want the HPV vaccine. Clinicians will need to remain alert to potentially vaccinating the entire family in the future.

Mid-adult women who considered it too late to receive the HPV vaccine were less likely to want it. In many cases, their assessment is probably correct, particularly for women 50 years of age and older. We did not specifically ask women why they thought it was too late. For many, this may be a false assumption. Simply having a history of an abnormal Papanicolaou test, or even surgery for cervical neoplasia, are not reasons to avoid vaccination. Similarly, a history of genital warts in a partner or themselves are not contraindications to being vaccinated. Other explanations may include same-sex relationships, sexual abstinence, and hysterectomy, but even some of these mid-adult women could potentially benefit from vaccination. 
Monogamous mid-adult women were also less likely to want to receive the HPV vaccine. Although these women perceive themselves not needing the vaccine, many may not remain with the same sexual partner their entire life because of divorce, separation, infidelity, or death of a spouse. Sexual infidelity by a spouse also places the unknowing monogamous mid-adult woman at risk for acquiring HPV.

Obviously, "catch-up" vaccination in mid-adult women poses many additional questions. However, this study has identified some useful correlates of HPV vaccine acceptance that contrast somewhat with independent predictors of Herpes simplex virus-2 vaccine acceptability (perceived benefits of vaccination, decreased exercise, and reduced alcohol consumption). ${ }^{9}$ Given information provided by this trial, clinicians will be better prepared to recognize mid-adult women who are receptive to or opposed to the HPV vaccine. Proper guidance should help reduce the risk for mid-adult women developing neoplasias of the lower genital tract.

\section{References}

1. Gardasil [Quadrivalent human papillomavirus (types 6,11,16 and 18) recombinant vaccine] prescribing information. Whitehouse Station (NJ): Merck and Co, Inc; 2006.

2. Ferris DG, Waller JL, Owen A, Smith J. Mid-adult women's attitudes about receiving the prophylactic human papillomavirus vaccine. J Low Genit Tract Dis $2007 ; 11: 166-72$.

3. Friedman AL, Shepeard H. Exploring the knowledge, attitudes, beliefs and communication preferences of the general public regarding HPV: findings from CDC focus group research and implications for practice. Health Educ Behav 2007;34:471-85. Epub 2006 September 22.

4. Davis K, Dickman ED, Ferris DG, Dias JK. Human papillomavirus vaccine acceptability among parents of 10- to 15-year-old adolescents. J Low Genit Tract Dis 2004;8:188-94.

5. Salmon DA, Moulton LH, Omer SB, DeHart MP, Stokley S, Halsey NA. Factors associated with refusal of childhood vaccines among parents of school-aged children: a case-control study. Arch Pediatr Adolesc Med 2005;159:470-6.

6. Omer SB, Pan WK, Halsey NA, et al. Nonmedical exemptions to school immunization requirements: secular trends and association of state policies with pertussis incidence. JAMA 2006;296:1757-63.

7. McClelland A, Liamputtong P. Knowledge and acceptance of human papillomavirus vaccination: perspectives of young Australians living in Melbourne, Australia. Sex Health 2006;3:95-101.

8. Brabin L, Roberts SA, Farzaneh F, Kitchener HC. Future acceptance of adolescent human papillomavirus vaccination: a survey pf parental attitudes. Vaccine 2006;24:3087-94. Epub 2006 February 9.

9. Zimet GD, Fortenberry JD, Fife KH, Tyring SK, Herne K, Douglas JM. Acceptability of genital herpes immunization. The role of health beliefs and health behaviors. Sex Transm Dis 1997;24:555-60. 